\title{
A MICROSCALE QUADRUPOLE MASS SPECTROMETER FOR ION SPECIES ANALYSIS
}

\author{
Satwik H. Deshmukh ${ }^{*}$, Lyon B. King ${ }^{\dagger}$, and Christopher R. Monroe \\ *Aerophysics, Inc., 30981, Woodbush Rd, Calumet, MI 49913 \\ ${ }^{\dagger}$ Michigan Technological University, Houghton, MI 49931 \\ * University of Michigan, Ann Arbor, MI 48109
}

\begin{abstract}
Investigators have identified a quadrupole structure that uses non-traditional geometry with rectangular cantilevered electrodes and that is amenable to semiconductor fabrication. Despite the revolutionary electrode geometry, the proposed microscale quadrupole mass filter displays a sizeable internal volume within which the field is equivalent to that of a conventional quadrupole mass filter with circular electrodes. Numerical analyses predict that the mass resolution and transmission efficiency are nearly identical with those of an equivalent cylindrical device. Batch processing techniques could be employed to produce massively parallel arrays of microscale quadrupole filters in a cost-effective manner. Prototype filters were fabricated using conventional semi-conductor manipulation protocols in conjunction with molecular beam epitaxy on GaAs/AlGaAs. The preliminary electric characterization of the prototype indicates broad mass range up to 1,500 amu with minimal power requirements. Such a micro device would be invaluable for light species analysis in numerous applications including plasma diagnostics, residual gas analysis, detection of chemical and products of biological agents, and integrated sensing of gas quality in medical and pharmaceutical applications.
\end{abstract}

\section{Introduction}

A typical quadrupole mass spectrometer (QMS), as shown in Figure 1, is formed from a set of four cylindrical electrodes placed between ion source and detector. Alternating combinations of DC and RF potentials are applied to opposite pairs of rods, which affect the transverse motion $(x$ - and $y$ - direction) of ions while the axial (z-direction) motion remains unaffected. The $x$ - and $y$-motions of an ion with mass $m$ and charge $e$ are described by canonical Mathieu equations according to

$$
\frac{d^{2} x}{d \varsigma^{2}}+(a-2 q \cos 2 \varsigma) x=0
$$

Eqn. $1.1 \frac{d^{2} y}{d \varsigma^{2}}-(a-2 q \cos 2 \varsigma) y=0$

$$
a=\frac{4 e U_{D C}}{m r_{0}^{2} \Omega^{2}}, q=\frac{2 e V_{R F}}{m r_{0}^{2} \Omega^{2}}, \Omega t=2 \varsigma
$$

where a and q are Mathieu parameters, $U_{D C}$ and $V_{R F}$ are the magnitudes of the $\mathrm{DC}$ and $\mathrm{RF}$ potentials, respectively, and $\Omega=2 \pi f$ is the RF drive frequency. $r_{0}$ is half the transverse separation of the electrodes at the point of closest approach. The Mathieu equations have stable solutions i.e. the ions with mass to charge ratio $\mathrm{m} / \mathrm{e}$ have stable trajectories, if the $a$ and $q$ parameters are within a

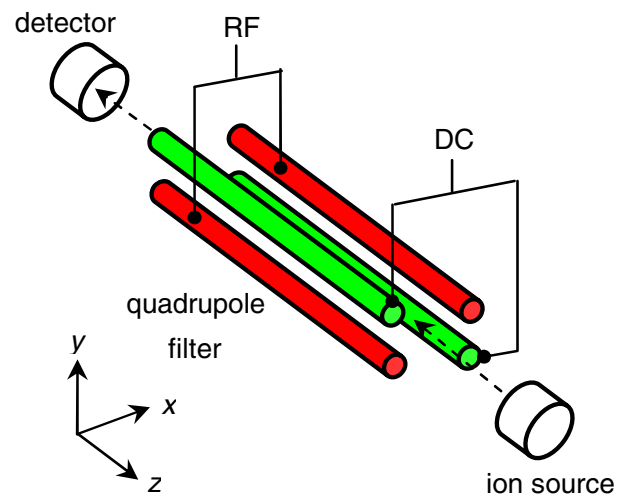

Figure 1. Schematic of a conventional linear quadrupole mass filter, showing DC (green) and RF (red) potentials applied to circular electrodes. The dashed arrows represent the ion trajectory axis through the filter.

specific range referred to as Paul's stability space. Hence $U_{D C}, V_{R F}$, and $\Omega$ are chosen such that the $x$ and $y$-motions of ions with a specific m/e only are stable through the quadrupole region i.e. they successfully travel from inlet to detector and are destructively measured as an electric current. All other ions are ejected in the transverse direction. A mass spectrum is achieved by scanning the electrode voltages in a manner that spans the ion mass range of interest. ${ }^{1}$ 


\subsection{Performance and Scaling Analysis for Quadrupole Mass Filter (QMF)}

Since the RF drive signal causes the transverse motion of undesired ions to become unstable, the mass resolution of a QMF is proportional to the number of RF cycles that the ions undergo prior to reaching the detector. The mass resolving capability (at 10\% peak height) of a traditional quadrupole filter in $\mathrm{kg}, \Delta m$, can be related to the RF drive frequency, $\Omega$, the axial energy of the source-ion in electron-volts, $V_{z}$, and the axial length of the quadrupole filter, $L, \mathrm{by}^{2}$

$$
\text { Eqn. 1.2 } \Delta m \approx \frac{40 e V_{z}}{(\Omega / \pi)^{2} L^{2}}
$$

To preserve transverse motion stability, the Mathieu parameters $a$ and $q$ must be invariant with physical scale. Holding $q$ (or $a$ ) constant, the resolution law from Eqn. 1.2 can be combined with the definition of $q$ in Eqn. 1.1 to show the relationship between the instrument resolution and physical parameters

$$
\text { Eqn. } 1.3 \quad \frac{\Delta m}{m} \propto \frac{r_{0}^{2} V_{z}}{V_{R F} L^{2}} .
$$

Realizing that the important parameter is not the RF voltage magnitude, but rather the strength of the electric field, $E_{R F} \sim V_{R F} / r_{0}$, the scaling criteria can be separated into terms reflecting ion source characteristics, applied transverse electric field, and filter geometry/size as follows

Eqn. 1.4

$$
\frac{\Delta m}{m} \propto V_{z} \frac{r_{0}}{L^{2}} \frac{1}{E_{R F}} .
$$

Since the method of ion formation (source) is governed by physics that does not scale (electron impact ionization, etc.), the value of $V_{z}$ will likely remain constant regardless of instrument size, probably equal to a few electron volts. Also, the value of $E_{R F}$ will likely be invariant on small devices, as the ability to apply voltage without breakdown is reduced with electrode spacing.

In terms of physical dimension, the resolution is dependent not on the aspect ratio, $r_{0} / L$, but instead depends more strongly on the axial length i.e. on $r_{0} / L^{2}$. This scaling behavior has important implications for fabricating a miniature quadrupole filter. Since the overall size of the instrument is given by the larger dimension, $L$, the transverse features, having characteristic dimension on the order of $r_{0}$, must be reduced in size quadratically with instrument size to preserve resolution. Thus, miniature quadrupoles must have aspect ratios much less than macroscopic counterparts, and the ability to manufacture electrodes with small transverse features will in turn limit the length reduction possible and hence the degree of miniaturization.

A typical macroscopic quadrupole mass filter employs cylindrical rods as electrodes with length $L \sim 10 \mathrm{~cm}$ and diameter $\sim 5 \mathrm{~mm}\left(r_{0} \sim 2.5 \mathrm{~mm}\right), \mathrm{RF}$ potential $V_{R F} \sim 1 \mathrm{kV}$, and source-ion axial energy $V_{z} \sim 10 \mathrm{eV}$ to achieve a mass resolution of $\mathrm{m} / \Delta \mathrm{m}$ $\sim 1000$. In order to reduce the largest dimension of the quadrupole filter by an order of magnitude from $10 \mathrm{~cm}$ to $1 \mathrm{~cm}$ while keeping resolution, $V_{z}$, and $E_{R F}$ constant, we require that the electrode spacing of the miniature instrument be $\sim 25 \mu \mathrm{m}$, yielding an instrument with an aspect ratio of 0.0025 , compared with 0.025 for the macro device. It is apparent that attempts to further reduce the largest dimension $L$ of the device much past $1 \mathrm{~cm}$ are quickly met with unreasonable reductions in electrode size and spacing, $r_{0}$.

\subsection{QMS Miniaturization Efforts}

While macroscopic QMS devices are widespread, attempts at drastic reduction in scale occurred only recently. Motivation for miniaturization of QMS sensors has come on two fronts: 1) Development of small instrumentation for spacecraft, and 2) Attempts to relax the vacuum requirements for residual gas analysis of ambient or near-ambient samples.

Orient, et. al., of the NASA Jet Propulsion Laboratory, fabricated a miniature QMS using conventional machining technology. ${ }^{3}$ The device utilized 2-mm-diameter electrodes of 25-mm length arranged in a $4 \times 4$ array. Employing a channel electron multiplier detector, $\Delta m$ of 0.1-0.5 amu was achieved. Similar to the Orient device, Holkeboer, et. al., utilized conventional fabrication methods (electrical discharge machining) to construct a QMS with 1-mm-diameter x 1-cm long rods. ${ }^{4}$ Using this device, Holkeboer, et. al., demonstrated QMS sampling at background pressures as high as 10 mTorr. The earliest available reference to functional QMS devices approaching micrometer dimensions is the instrument developed by Taylor, et. al. Again motivated by spacecraft miniaturization trends, Taylor, et. al. blended silicon-based fabrication techniques with manual assembly techniques to construct a QMS with 0.5-mm-diameter electrodes of 20-30-mm length. ${ }^{5}$ The Taylor, et. al. 
instrument utilized metal coated optical fibers as electrodes; these electrodes were then bonded to etched grooves in a silicon substrate to form the filter region. The first published reference of a mass spectrometer manufactured exclusively using "on-a-chip" technology is the $E x B$ Wien-type filter reported by Sillon and Baptist. ${ }^{6}$ For their Wien filter, Sillon and Baptist utilized a complete allsilicon fabrication process to construct individual velocity-selecting channels with electrode transverse dimensions of $200 \mu \mathrm{m}$. Although a micro-machined quadrupole filter is mentioned in the journal article, no published reference to this device could be located.

\section{Microscale Quadrupole Mass Filter with Cantilevered Rectangular Electrodes}

\subsection{Micro Quadrupole ( $\mu$ Quad) Electrode Geometry}

The scaling analysis in section 1.1 shows that the QMS miniaturization efforts are mainly dictated by the ability to fabricate and align miniature electrodes. The foundation for the research presented here is the innovative micro quadrupole electrode geometry developed by Monroe's quantum computing research group at the University of Michigan ${ }^{7}$. The structure is a significant departure from conventional design wisdom. A schematic of the proposed $\mu \mathrm{Quad}$ structure is shown in Figure 2. Departing from the cylindrical geometry of conventional macro devices, the $\mu Q$ uad employs rectangular electrodes. The rectangular geometry enables extreme miniaturization of transverse features by costeffective semiconductor fabrication techniques. The $\mu Q$ uad electrodes can be fashioned from $\mathrm{Si}$ doped GaAs conductors separated by insulating layers of $\mathrm{Al}_{0.7} \mathrm{Ga}_{0.3}$ As. By cantilevering the electrodes out from the insulating layer, electric field distortion arising from insulator charging is made negligible within the filter region. The electrode layer separation is denoted by $h$, while the horizontal gap between electrodes is denoted by $w$. Filter radius $r_{0}$ is given by $\left[(h / 2)^{2}+(w / 2)^{2}\right]^{1 / 2}$. The transverse aspect ratio $\alpha$ is defined as the ratio of horizontal gap between electrodes to electrode layer separation, i.e. $w / h$. Since the long dimension, $L$ is in the plane of the substrate and $r_{0}$ is on the order of a few microns, fabrication of elements with very small $r_{0} / L$ ratio is possible even for small

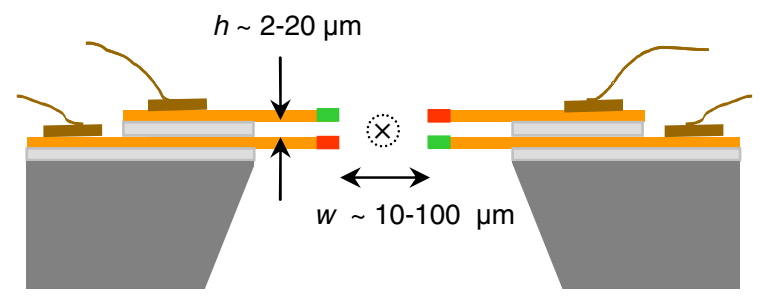

Figure 2. Axial view of a $\mu$ Quad element (not to scale). The ion axis ( $z$-direction) is indicated by the " $x$ " symbol. The equivalent of the four rods of the $\mu$ Quad are provided by the edges of cantilevered electrodes carrying DC (green) and RF(red) potentials, and giving rise to the effective confinement volume approximately indicated by the circular dotted line. The light grey spacers are insulating barriers, and the bottom dark dray layer is the substrate.

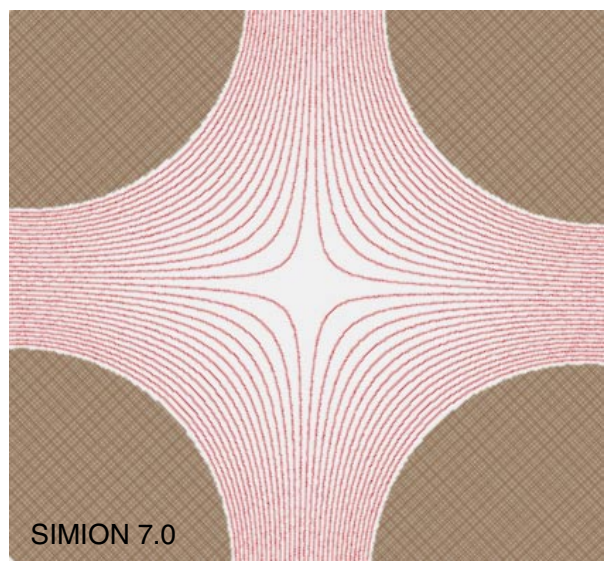

(a)

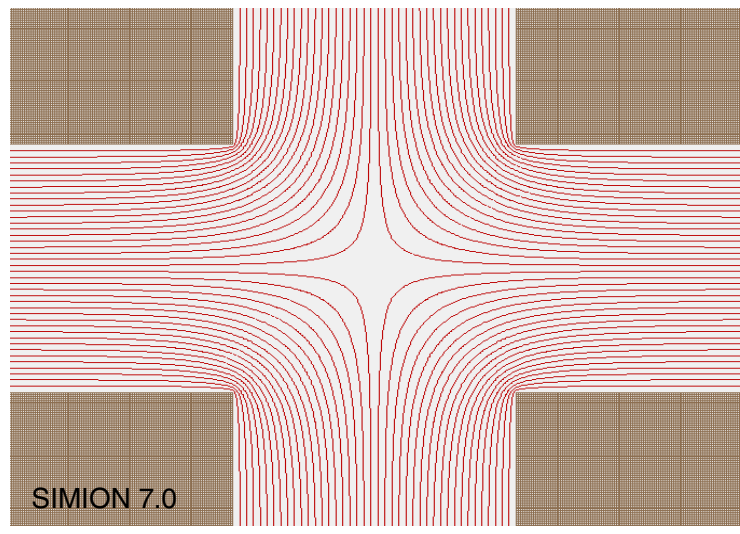

(b)

Figure 3. Comparison of electric equipotential lines within (a) a classic quadrupole with cylindrical electrodes, and (b) a $\mu$ Quad with novel rectangular electrode geometry (electrodes are shown as cross-hatched areas in brown color).

L. Despite the revolutionary geometry, the proposed $\mu Q$ uad displays a sizeable internal 
volume within which the field is equivalent to a conventional quadrupole with cylindrical electrodes. Figure 3 demonstrates the similarity between the $\mu$ Quad field and a conventional cylindrical quadrupole field. Detailed numerical studies have been performed that indicate less than $0.1 \%$ difference between the fields in the central region of the filter. This is noteworthy, as the rectangular geometry can be produced at the micron-level using semiconductor techniques, avoiding the need to fabricate and align microsize cylinders. While $\mu \mathrm{Quad}$ structures with aspect ratios $(\alpha)$ higher than 1 may be easier to fabricate, layer separation $h$ is the dimension that decides the field region that is equivalent to a conventional quadrupole field. ${ }^{7}$

\section{2. $\mu$ Quad Simulation Testbed}

In order to gauge the utility of a $\mu$ Quad as a quadrupole mass filter, numerical simulations were performed using SIMION 7.0 software to compute electric field and to predict the ion trajectories within a model $\mu$ Quad. The stability diagram or operating space of the model $\mu$ Quad was mapped and its performance was assessed in terms of ion transmission efficiency and mass resolution for realistic geometric and operating parameters. Investigators developed a custom code, Transmission and Mass Resolution Calculator (TMRC), to interface with SIMION's trajectory simulations that incrementally varied search parameters to systematically explore the $\mu \mathrm{Quad}$ stability space.

Axial view of the 3D computational model of $\mu$ Quad is shown in Figure 4. The geometric parameters of the model were based on the scaling relations discussed in Section 1.1 and manufacturability constraints with the goal of making a $\mu$ Quad with resolution comparable to that of a macroscale cylindrical quadrupole. A practical value of $8 \mu \mathrm{m}$ for layer thickness $t$ and layer separation $h$, and transverse aspect ratio of 2 were chosen from manufacturing point of view. $r_{0}$ can be calculated from the layer separation $h$ and the aspect ratio and amounts to be $8.944 \mu \mathrm{m}$. Using the scaling relations and with some initial trial and error tests, the length $L$ of the $\mu$ Quad was set at 1.7 $\mathrm{cm}$ to obtain $m / \Delta m=1000$. The ion energy $V_{z}$, was kept constant at a typical value of $5 \mathrm{eV}$ for all the ions injected during the simulations. Assuming that the $\mu \mathrm{Quad}$ will behave similar to a conventional quadrupole, the performance scaling relation of Eqn. 1.2 was used to establish the operation frequency for the given dimensions and desired resolution at $83 \mathrm{MHz}$. All simulations described in this section were performed for singly charged test ions with nominal mass of 100 amu. For simplicity of analyses, ions were injected exactly from the $x-y$ plane at the inlet of the filter and the effect of fringing fields was not considered.

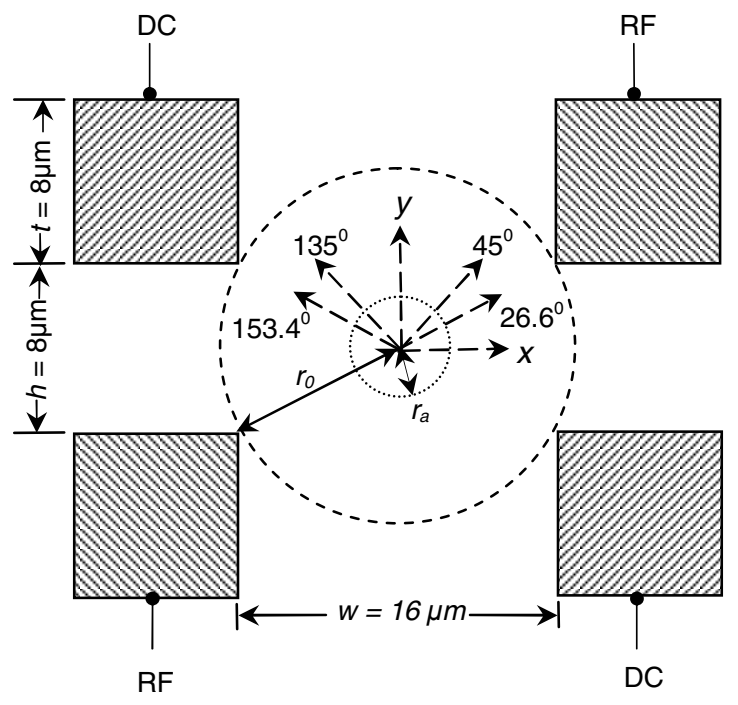

Figure 4. Axial view ( $x-y$ plane) of the simulation model of the $\mu$ Quad with $\alpha=2$. The electrodes are shown by hatched areas. z-axis is at the center of the filter and is directed into the plane of the paper. The length of the mass filter in the $z$-direction is $17 \mathrm{~mm}$. The azimuth angles at which test ions were injected are shown by dashed arrows. The approximate acceptance aperture of the $\mu$ Quad at a sample operating $(a-q)$ point is shown by dotted circle.

\section{3. $\mu$ Quad Transmission and Mass Resolution Simulations}

In the case of quadrupole mass filter with hyperbolic electrodes the correct values of $V_{\mathrm{RF}}$ and $U_{D C}$ for a desired mass resolution and transmission efficiency can be obtained from the well known Paul's stability diagram [see Figure 12] as points in $a-q$ space. As the electric field in $\mu$ Quad differs from that of a conventional quadrupole, the values of $q$ and $a$, and in turn the RF and DC voltages to stabilize the $100 \mathrm{amu}$ ion (or the tuned mass) will be different from those from the Paul's stability diagram. Through the numerical simulations the RF and DC potentials of the rectangular electrodes were varied. The range of these voltages at which the target mass of $100 \mathrm{amu}$ is stable was mapped in terms of dimensionless parameters $a$ and $q$ that can be defined as in Eqn. 1.1. 
The procedure to map the stability diagram for a $\mu$ Quad is summarized here. It will be convenient to describe the concept of acceptance aperture $A_{a}$ at this point. Acceptance aperture area $A_{a}$, is a circular region in $x-y$ plane located at the entrance of a mass filter and centered at the filter axis. Let us assume that the mass filter is tuned for a certain target mass of $m$ amu. Then all $m$ amu ions entering parallel to the mass filter axis have $100 \%$ transmission efficiency if their entrance position is within the acceptance aperture. The radius of this acceptance aperture is denoted by $r_{a}$. Ions injected into the filter region at radial positions greater than $r_{a}$, while mathematically stable, will have transverse oscillations of sufficient amplitude to cause them to impact the electrodes or be lost from the filter region. Thus, $A_{a}$ represents the "usable" region of the filter and, hence, determines the transmission efficiency.

A stable operating point was defined as a set of $a$ and $q$ (or $V_{R F}$ and $U_{D C}$ ) that permitted a tunedmass ion to pass through the $\mu$ Quad when injected parallel to the filter axis at some initial radial position $r$ that is greater than zero (all ions injected parallel to the mass filter and exactly at $r=0$ will pass as this is a field null). In terms of acceptance radius, stable operating point is defined as any operating point where $r_{a}>0$ (finite transmission). It is thus possible to simultaneously map the stability diagram and determine the acceptance radius by determining $r_{a}$ throughout the $a-q$ space and identifying the boundary where $r_{a}$ goes to zero. A representation of this stability space is indicated in Figure 5.

An initial search region within the $a-q$ space was found by trial and error method with large values of $i$ and $j$ (coarse $a-q$ resolution) as shown in the Figure 5. As simulations concluded and stability boundaries were found from the broad search, a subspace within the original $a-q$ search space containing the peak of the stability region was identified and the procedure was repeated in this subspace with increasing resolution in the $a-q$ grid. The goal was to accurately locate and characterize the $\mu$ Quad performance where mass resolution is expected to be the greatest.

The value of $r_{a}$ for a given $a-q$ point was determined by systematically injecting ions into the filter at different radial positions and determining whether the injected ions successfully passed through the filter region to the exit without impacting an electrode. The radial space was searched using a bracketing method. An initial tuned-mass ion was injected at a radial position of $r=r_{0} / 2$ (midway between filter axis and electrode tip). If this ion successfully passed through the filter, the next ion was injected halfway between the successful position and the electrode tip i.e. at $r=3 r_{0} / 4$. If the initial ion was unsuccessful, the second ion was instead injected at $r=r_{0} / 4$. Using this method to establish upper and lower bounds

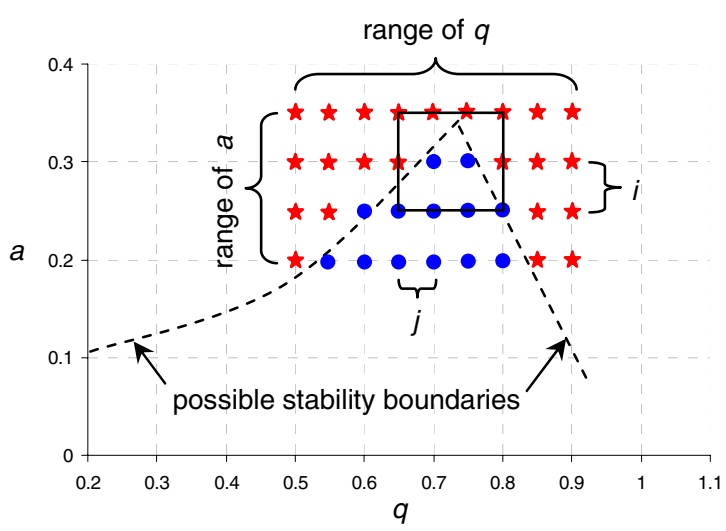

Figure 5. A sample $a-q$ search space in which the acceptance aperture radius $r_{a}$ was calculated at each operating point denoted by red stars and blue dots, in order to map the stability diagram for the $\mu$ Quad model. For blue dot-operating points $r_{a}>0$, hence they constitute the stability region while red star-operating points lie outside the stability region. The $a-q$ subspace with increased resolution for next iteration of simulations in the process of mapping the stability region is indicated by a rectangle.

for $r_{a}$, the halving procedure was repeated until the upper bound of successful radial position was within one model grid spacing $(0.01 \mu \mathrm{m})$ of the lower bound. The acceptance radius $r_{a}$ was then defined as this radial distance of injection.

The ion trajectory stability is dependent not only on the radius of injection, but also on the azimuthal angle of the initial ion position (initial RF phase angle $)^{8}$. To capture this effect, the bracketing procedure to find $r_{a}$ described above was conducted at six azimuth angles: 0, 26.6,45, 90,135 , and 153.4 degrees considering the symmetry of the quadrupole field, as shown in Figure 4. The acceptance radius, $r_{a}$, was then defined as the largest radial distance of injection $r$ for which ions at all above azimuth angles pass through the filter.

The TMRC code concurrently calculated the $\mu$ Quad mass resolution during the stability/acceptance aperture simulations. For each $a-q$ point simulated at which $r_{a}>0$, the TMRC code systematically injected ions of varying mass to determine the minimum and maximum mass ion that successfully passed through the filter and, thus, $\Delta m$. Once $r_{a}$ was determined, the mass search was performed by injecting ions at a radial distance of 


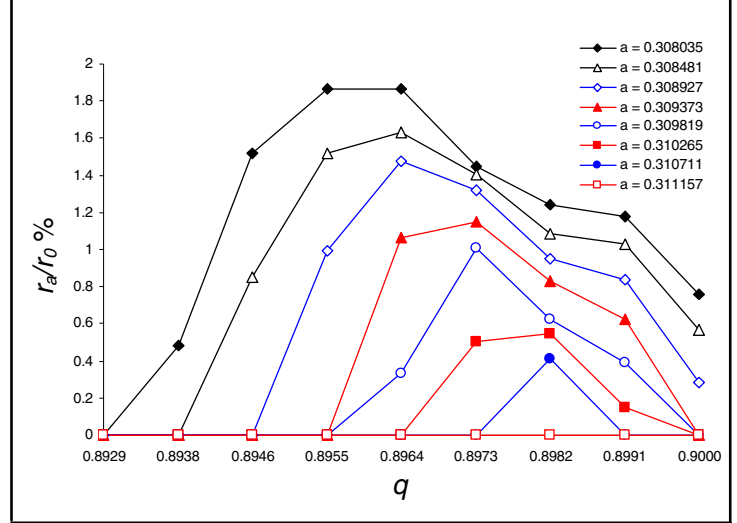

Figure 6. Variations in the radius of acceptance aperture $r_{a}$ expressed as a percentage of mass filter radius $r_{0}$, for $\mu$ Quad. Data represents the region near the tip of the stability space.

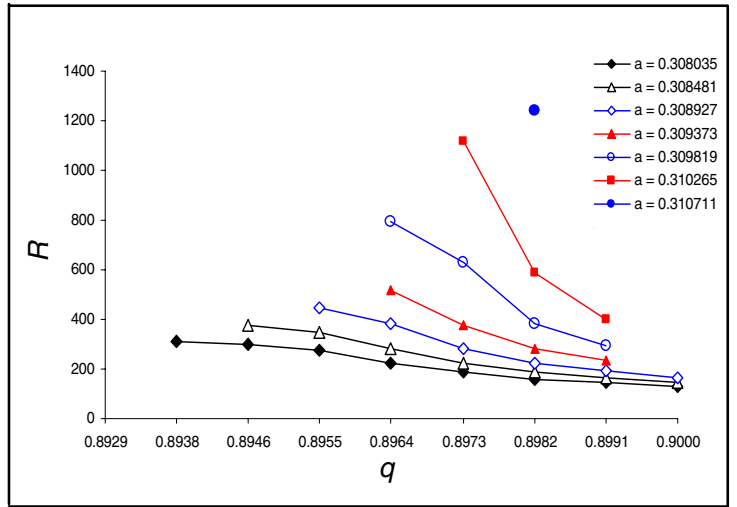

Figure 7. Variations in the mass resolution $R$ for $\mu$ Quad. Data represents the region near the tip of the stability space.

$r_{a} / 2$ (for all azimuth angles as described above). Since the code had already determined that the tuned mass ion (100 amu in this case) was transmitted through the filter, the goal was to identify the upper and lower mass limits surrounding the tuned mass. To determine the lower mass limit, an initial ion of sufficiently low mass (e.g. $m=1 \mathrm{amu}$ ) was injected. If this ion successfully passed through the filter, a second ion with a mass of one-half the initial ion mass was subsequently tracked. If the initial ion was unsuccessful, a second ion with mass mid-way between the initial mass and the tuned mass was injected. This bisection of the mass range was repeated until the lower mass limit was found to be within $0.01 \mathrm{amu}$ of the previously tested ion mass. A similar bracketing procedure determined the upper mass limit. The mass range, $\Delta m$, was defined as half the width of the mass window i.e.
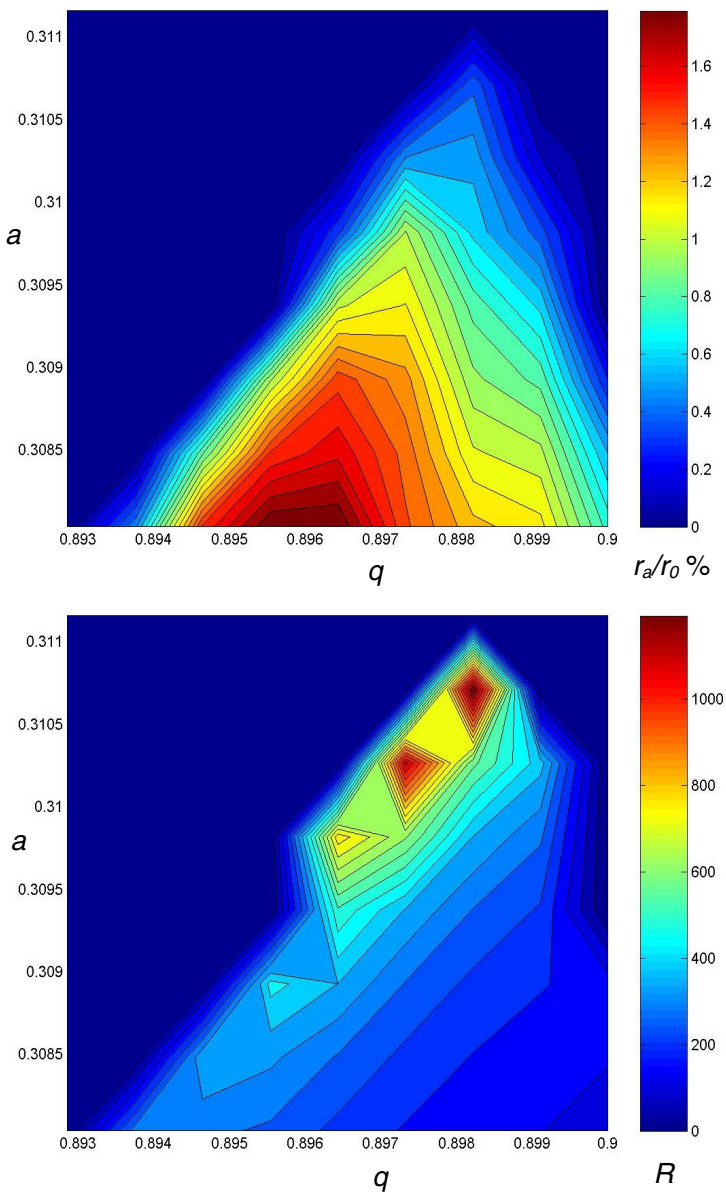

Figure 8. Design space for $\mu \mathrm{Quad}$

the difference between upper and lower mass limits. The resolution $R$ was defined as the ratio of tuned mass $m$ to the mass range $\Delta m$.

Numerous $a-q$ subspaces were searched to locate the stability peaks of the $\mu$ Quad following the method shown in the Figure 5. Since it is known that acceptance aperture (transmission) varies inversely with mass resolution, the search focused on filter behavior at high mass resolution (small $r_{a}$ ). Only the results for final search space with highest resolution are presented here. Figure 6 shows the acceptance radius, $r_{a}$, expressed as a percentage of the filter size, $r_{0}$, near the peak of the stability diagram. The various curves in the figure denote varying values of $a$, with $q$ expressed on the horizontal axis. The same search spaces used to find the acceptance radius were used for the resolution calculations. Figure 7 shows the mass resolution for $\mu \mathrm{Quad}$ operated in the same stability region as that of Figure 6, and having mass resolution comparable with conventional cylindrical quadrupole. 


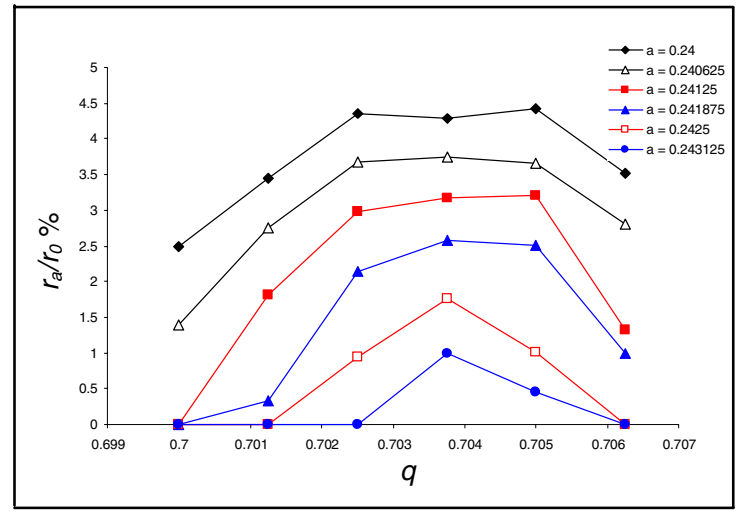

Figure 9. Acceptance aperture radius calculated by TMRC for a conventional cylindrical quadrupole mass filter (geometrically equivalent to the $\mu$ Quad) operating near peak of stability diagram.

The trade-off between resolution and transmission can best be analyzed for the $\mu \mathrm{Quad}$ by consolidating the performance within the $a-q$ space of interest. Contour plots were constructed, wherein $a$ and $q$ vary along the plot axes, and acceptance aperture/resolution are represented by color contours. Such plots were compiled for all $a$ $q$ search spaces; only the plots for operation region of interest (near the peak) are shown in Figure 8. It is apparent from these plots that there are well defined regions in the $a-q$ space where the acceptance aperture radius $r_{a}$ is on the order of a few percent of $r_{0}$, while the mass resolution is on the order of 1,000 or greater.

\subsection{Comparison of $\mu Q u a d$ with Equivalent Conventional Cylindrical Quadrupole}

While the stability space, resolution, and transmission of conventional cylindrical quadrupole are well established experimentally and analytically in the literature, it may be misleading to compare such values directly to the numerical simulations performed for the $\mu$ Quad, as artifacts of the simulation may affect the results. Investigators, therefore, used the TMRC code to establish the stability behavior, transmission, and mass resolution of a conventional cylindrical quadrupole that is equivalent to the $\mu$ Quad device described in Section 2.2. As layer separation $h$ of the $\mu$ Quad decides "usable region" within the filter, the distance between opposite pairs of electrodes i.e. $2 r_{0}$ of the equivalent cylindrical quadrupole was kept at $\left(2 h^{2}\right)^{1 / 2}$. Singly charged 100 amu ions were subjected to the same number of RF cycles within the filter, as in case of the $\mu$ Quad, by keeping the filter length $L$, ion energy $\mathrm{V}_{\mathrm{z}}$, and RF frequency $\Omega$ constant. The acceptance radius, mass

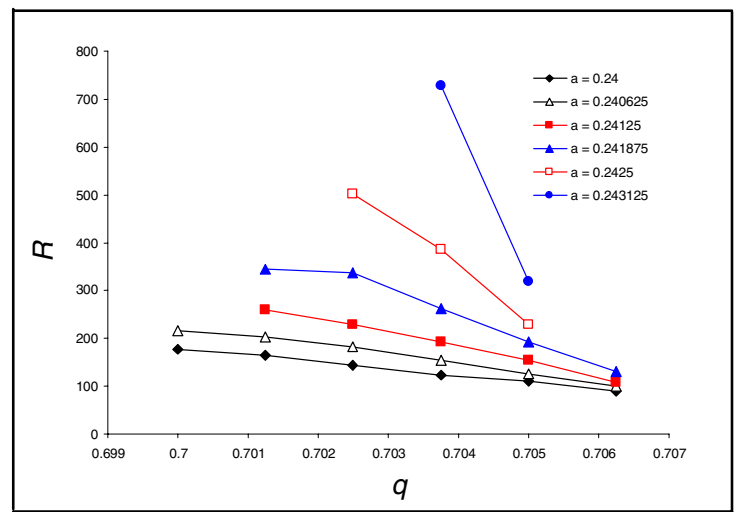

Figure 10. Mass resolution $R$ calculated by TMRC for a conventional cylindrical quadrupole mass filter (geometrically equivalent to the $\mu$ Quad) operating near peak of stability diagram
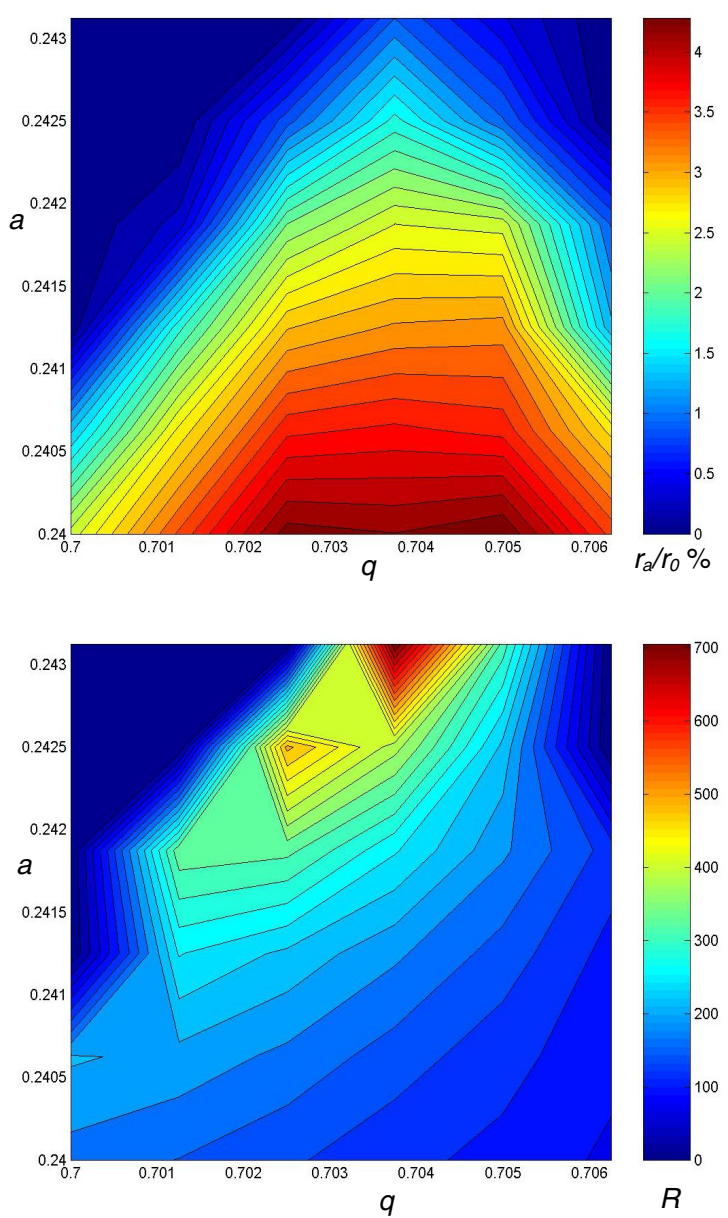

Figure 11. Design space for conventional cylindrical quadrupole mass filter (geometrically equivalent to the $\mu Q u a d)$ operating near peak of stability diagram 
resolution, and design- space analysis for the equivalent cylindrical quadrupole are presented in Figure 9, Figure 10, and Figure 11.

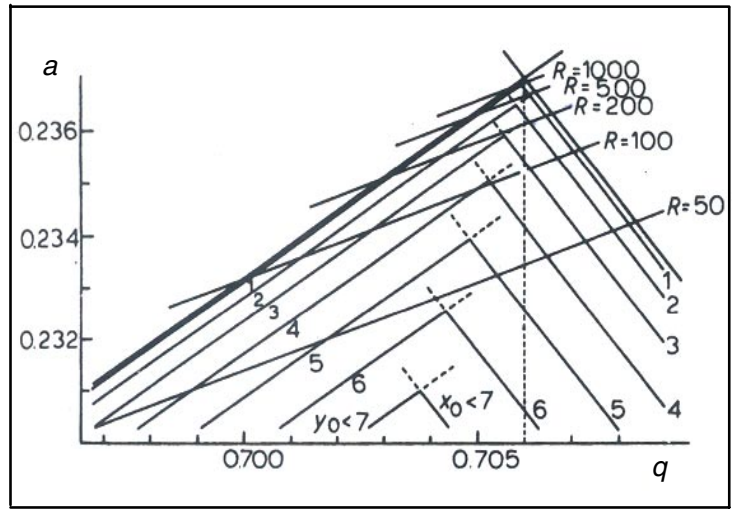

Figure 12. The tip of the Paul's stability diagram showing operating lines nominally giving resolutions of 50,100 , 200, 500 and 1000 for mass filter with hyperbolic electrodes. The lines parallel to the stability boundaries represent the maximum allowable initial $x$ and $y$ displacements (denoted as $x_{0}$ and $y_{0}$ ) expressed as a percentage of filter radius $r_{0}$, if there is to be $100 \%$ ion transmission for all initial RF phases. The calculations were made for ion entry parallel to the filter axis and in the absence of fringing fields.

With some confidence, then, that the TMRC is producing realistic results, the design space analyses for the $\mu$ Quad can be compared with those for a conventional cylindrical quadrupole. By comparing the color contour plots of both of them, it can be seen that the mass resolution and transmission behavior of the $\mu$ Quad is very similar to that of a conventional quadrupole. Thus, the scaling arguments presented in this report seem to be valid, and the construction of a $\mu$ Quad with dimensions shown here, yet having performance comparable with a conventional cylindrical quadrupole, seems feasible. For a $\mu$ Quad, it should be possible to achieve mass resolution near 1,000 with acceptance apertures a few percent of $r_{0}$.

\section{3. $\mu$ Quad Prototype Fabrication and Electrical Characterization}

The investigators have fabricated a simple prototype $\mu$ Quad based on gallium arsenide (GaAs) and aluminum gallium arsenide (AlGaAs) structures, using advanced molecular beam epitaxy (MBE), lithographic, and plasma etching techniques. The electron micrographs of this prototype are shown in Figure 13. The key feature
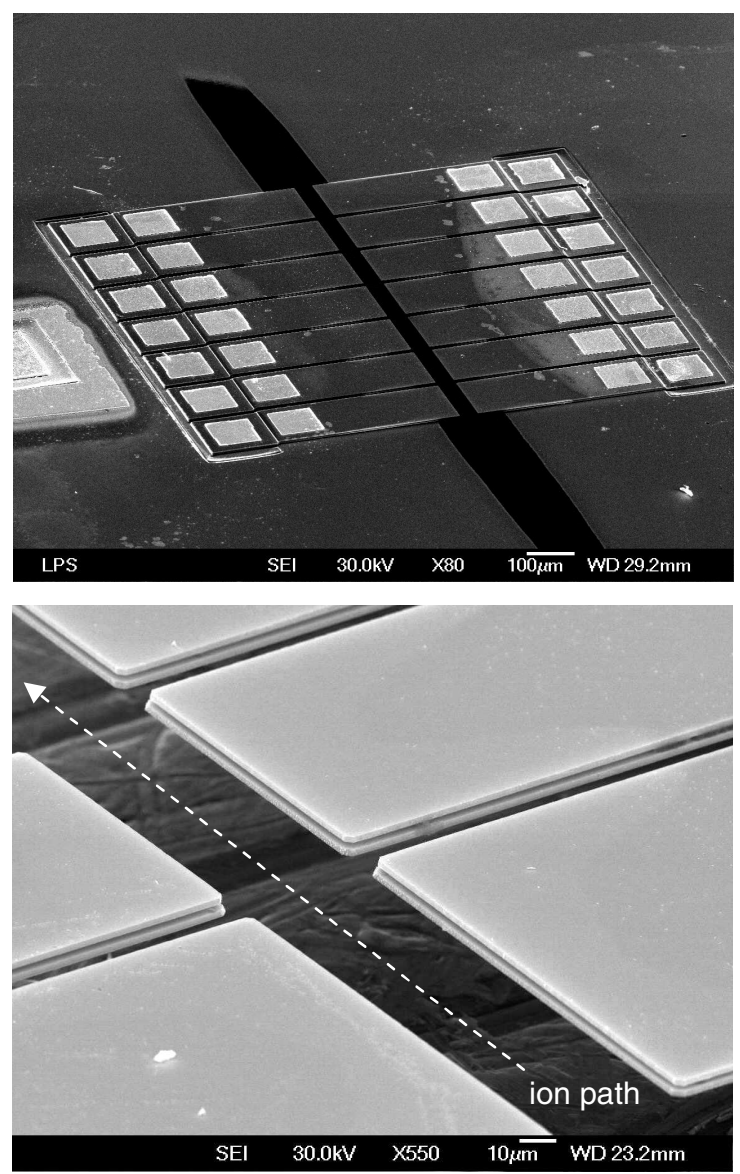

Figure 13. Electron micrograph of GaAs/AIGaAs prototype $\mu$ Quad. (top) Overview of substrate from top, with cantilevered electrodes in middle of figure. (bottom) Closeup of cantilevered electrodes, with clear 2 micron void between upper and lower GaAs electrodes where AIGaAs has been removed. The horizontal gap (dimension $w$ in Figure 2) is $40 \mu \mathrm{m}$.

of the proposed design is that all electrodes and insulating spacers are integral components, with no electrode "assembly" required. The excellent relative alignment of the electrodes follows from the tolerances of the lithographic etching techniques. In the initial prototype layer thickness $t$, and layer separation $h$ were chosen to be $2 \mu \mathrm{m}$ as thicker layers are costly and more time consuming. The electrode spacing $w$ was chosen to be $40 \mu \mathrm{m}$ for manufacturing convenience. Once the electrodes and insulating spacers have been fabricated, bonding pads were grown onto the outsides of the electrodes that can be seen (in white color) in top picture in Figure 13. These pads consist of alternating layers of germanium and gold, and are sufficiently tough so that conventional wire-bonding procedures permit gold ribbon to be firmly attached to the electrodes for electrical characterization. The electrodes were 
segmented axially into seven sections during the fabrication procedure to demonstrate the ability to fabricate geometrically complex structures.

In preliminary experimental testing voltages up to 5 volts at $25 \mathrm{MHz}$ were successfully applied to the prototype with no problems encountered. For the microscale dimensions considered in this study, the successfully applied voltage would permit detection of ions with masses as large as 1,500 amu. We anticipate that future designs with thicker layers, higher layer separation, and lower aspect ratio should allow order-of-magnitude higher potentials without compromising the $\mu \mathrm{Quad}$ mass selectivity and ion stability. Calculations indicate that the structures should be able to accommodate voltages up to about 50 volts before failure, hinting at a broad range of detectable masses.

\section{Conclusions}

While manufacturing microscale quadrupole mass filter, transverse features having characteristic dimension on the order of $r_{0}$, must be reduced in size quadratically with instrument length to maintain the resolution comparable to that of macroscopic devices (about $m / \Delta m \sim 1000$ ). Hence ability to manufacture microscale transverse features will limit the length reduction possible and in turn the degree of miniaturization.

Numerical simulations indicated that $\mu \mathrm{Quad}$ displays mass resolution and transmission efficiency nearly identical to that demonstrated by equivalent cylindrical quadrupole. The operating space of $\mu$ Quad clearly shows a subspace in which the mass resolution was on the order of 1,000 while the acceptance radius was a few percent of the filter size, $r_{0}$. This implies that the ion beam throughput and, hence, the sensitivity of $\mu$ Quad can be scaled directly from existing experimental and analytic data for conventional cylindrical quadrupole. Since the sensitivity (ion signal throughput) of a quadrupole filter scales with the square of its transverse dimension, the ratio of sensitivity of a $\mu$ Quad to that of a macro-Quad is $\mathrm{I}_{\text {micro }} / \mathrm{I}_{\text {macro }} \sim(25 \mu \mathrm{m} / 2.5 \mathrm{~mm})^{2}=1 \times 10^{-4}$. Using batch processing techniques, the $\mu$ Quad elements could be combined into massively parallel arrays in a cost-effective manner. Up to 10,000 individual $\mu$ Quad filters could be arranged in a $1 \mathrm{~cm}^{3}$ structure and operated to select the same mass. Such a parallel device will have sensitivity ratio of $\mathrm{I}_{\text {micro }} / \mathrm{I}_{\text {macro }} \sim 1 \times 10^{-4} \times 10,000=1$. Thus the resulting micro-device could exhibit sensitivity and mass selectivity $(m / \Delta m \sim 1000)$ comparable with macroscale device while occupying only $1 \mathrm{~cm}^{3}$.

Prototype micro-cantilevered electrode structures were fabricated using conventional semiconductor manipulation protocols in conjunction with $\mathrm{MBE}$ on $\mathrm{GaAs} / \mathrm{AlGaAs}$. In the preliminary electric characterization voltages up to 5 volts at 25 $\mathrm{MHz}$ were successfully applied with no problems encountered. The power dissipation was as minimal as $20 \mathrm{~mW}$. For the prototype dimensions considered in this study, $5 \mathrm{~V}$ RF at $25 \mathrm{MHz}$ would correspond to a tuned-mass ion of 1,500 amu. Calculations indicate that the structures should be able to accommodate voltages up to about $50 \mathrm{~V}$ before failure, hinting at a broad mass range.

\section{Acknowledgements}

Work reported here was supported by the National Institute of Standards and Technology under a Phase I contract. This support is gratefully acknowledged.

\section{References}

${ }^{1}$ Dawson, P.H., Quadupole Mass Spectrometry and Its Applications, American Institute of Physics, pp. 9-78 (1995)

${ }^{2}$ Austin, W.E., Home, A.E., and Leck, J.H., Quadrupole Mass Spectrometry and Its Applications, AIP Press, New York (1995).

${ }^{3}$ Orient, O.J., Chutjian, A., and Garkanian, V., Rev. Sci. Instrum. 68, 3, pp. 1393-1397 (1997).

${ }^{4}$ Holkeboer, D.H., Karandy, T.L., Currier, F.C., Frees, L.C., and Ellefson, R.E., J. Vac. Sci. Technol. A 16, 3, pp. 1157-1162 (1998).

${ }^{5}$ Taylor, S., Tindall, R.F., and Syms, R.R.A., J. Vac. Sci. Technol. B 19, 2, pp. 557-562 (2001).

${ }^{6}$ Sillon, N., and Baptist, R., Sensors and Actuators B 83, pp. 129-137 (2002).

${ }^{7}$ Madsen, M.J., Hensinger, W.K., Stick, D., Rabchuk, J.A., Monroe, C., Appl. Phys. B, DOI: 10.1007/s00340-004-1414-9 (2004)

${ }^{8}$ Ma, F.A., Taylor, S., IEE Proc.-Sci. Meas. Technol. 143, 1, pp. 71-76 (1996). 